\title{
Separability of Real Normed Spaces and Its Basic Properties
}

\author{
Kazuhisa Nakasho \\ Shinshu University \\ Nagano, Japan
}

\author{
Noboru Endou \\ Gifu National College of Technology \\ Gifu, Japan
}

Summary. In this article, the separability of real normed spaces and its properties are mainly formalized. In the first section, it is proved that a real normed subspace is separable if it is generated by a countable subset. We used here the fact that the rational numbers form a dense subset of the real numbers. In the second section, the basic properties of the separable normed spaces are discussed. It is applied to isomorphic spaces via bounded linear operators and double dual spaces. In the last section, it is proved that the completeness and reflexivity are transferred to sublinear normed spaces. The formalization is based on [34, and also referred to [7], 14] and [16].

MSC: 46B20 46A19 03B35

Keywords: functional analysis; normed linear space; topological vector space

MML identifier: NORMSP_4, version: 8.1.04 5.31.1231

The notation and terminology used in this paper have been introduced in the following articles: [2], 44, 88, [26], [20], [21], [13], [9], 10], 22], [1], 25], [24], [15], [19], 6], [1], 23], [17, 32], [33], 27], 28], 29], [30], 31], [18], and [12].

\section{Separability of Real Normed Space}

Let $X$ be a real linear space and $A$ be a subset of $X$. The functor Sums $A$ yielding a subset of $X$ is defined by the term

(Def. 1) $\left\{\sum l\right.$, where $l$ is a linear combination of $\left.A: \operatorname{rng} l \subseteq \mathbb{Q}\right\}$.

Let us consider a real normed space $V$ and a real normed subspace $V_{1}$ of $V$. Now we state the propositions: 
(1) TopSpaceNorm $V_{1}$ is a subspace of TopSpaceNorm $V$.

Proof: For every points $x, y$ of MetricSpaceNorm $V_{1}$, (the distance of MetricSpaceNorm $\left.V_{1}\right)(x, y)=($ the distance of MetricSpaceNorm $V)(x, y)$ by [28, (16)], [19, (28)].

(2) LinearTopSpaceNorm $V_{1}$ is a subspace of LinearTopSpaceNorm $V$. The theorem is a consequence of (1).

Now we state the proposition:

(3) Let us consider a real normed space $X$, and real normed subspaces $Y, Z$ of $X$. Suppose there exists a subset $A$ of $X$ such that $A=$ the carrier of $Y$ and $\bar{A}=$ the carrier of $Z$. Let us consider a subset $D_{0}$ of $Y$, and a subset $D$ of $Z$. If $D_{0}$ is dense and $D_{0}=D$, then $D$ is dense.

Proof: LinearTopSpaceNorm $Z$ is a subspace of LinearTopSpaceNorm $X$ and LinearTopSpaceNorm $Y$ is a subspace of LinearTopSpaceNorm $X$. For every subset $S$ of $Z$ such that $S \neq \emptyset$ and $S$ is open holds $D$ meets $S$ by [15, (16), (20)], [19, (5), (17), (4)].

Let us consider an additive loop structure $X$ and subsets $A, B$ of $X$. Now we state the propositions:

(4) There exists a function $F$ from $A+B$ into $A \times B$ such that $F$ is one-toone.

Proof: Set $D=A+B$. Define $\mathcal{P}$ [object, object $] \equiv$ there exist points $a, b$ of $X$ such that $\$_{1}=a+b$ and $a \in A$ and $b \in B$ and $\$_{2}=\langle a, b\rangle$. For every object $x$ such that $x \in D$ there exists an object $y$ such that $y \in A \times B$ and $\mathcal{P}[x, y]$ by $[12$, (87)]. Consider $F$ being a function from $D$ into $A \times B$ such that for every object $x$ such that $x \in D$ holds $\mathcal{P}[x, F(x)]$ from [10, Sch. 1]. For every objects $x_{1}, x_{2}$ such that $x_{1}, x_{2} \in \operatorname{dom} F$ and $F\left(x_{1}\right)=F\left(x_{2}\right)$ holds $x_{1}=x_{2}$.

(5) If $A$ is countable and $B$ is countable, then $A+B$ is countable. The theorem is a consequence of (4).

Now we state the proposition:

(6) Let us consider a non empty additive loop structure $X$, subsets $A, B$ of $X$, a linear combination $l_{1}$ of $A$, and a linear combination $l_{2}$ of $B$. Suppose $A$ misses $B$. Then there exists a linear combination $l$ of $A \cup B$ such that

(i) the support of $l=\left(\right.$ the support of $\left.l_{1}\right) \cup\left(\right.$ the support of $\left.l_{2}\right)$, and (ii) $l=l_{1}+l_{2}$.

Proof: Define $\mathcal{P}$ [object, object] $\equiv$ if $\$_{1} \in$ the support of $l_{1}$, then $\$_{2}=$ $l_{1}\left(\$_{1}\right)$ and if $\$_{1} \in$ the support of $l_{2}$, then $\$_{2}=l_{2}\left(\$_{1}\right)$ and if $\$_{1} \notin$ the support of $l_{1}$ and $\$_{1} \notin$ the support of $l_{2}$, then $\$_{2}=0$. Consider $l$ being a function from the carrier of $X$ into $\mathbb{R}$ such that for every object $x$ such 
that $x \in$ the carrier of $X$ holds $\mathcal{P}[x, l(x)]$ from [10, Sch. 1]. Reconsider $T=$ (the support of $\left.l_{1}\right) \cup\left(\right.$ the support of $l_{2}$ ) as a finite subset of $X$. For every element $x$ of $X$ such that $x \notin T$ holds $l(x)=0$. For every element $v$ of $X, l(v)=l_{1}(v)+l_{2}(v)$.

Let us consider a non empty additive loop structure $X$, subsets $A, B$ of $X$, and a linear combination $l$ of $A \cup B$. Now we state the propositions:

(7) There exists a linear combination $l_{1}$ of $A$ such that

(i) the support of $l_{1}=$ (the support of $\left.l\right) \backslash B$, and

(ii) for every element $x$ of $X$ such that $x \in$ the support of $l_{1}$ holds $l_{1}(x)=$ $l(x)$.

Proof: Reconsider $T_{1}=$ (the support of $l$ ) \B as a finite subset of $X$. Define $\mathcal{Q}$ [object, object] $\equiv$ if $\$_{1} \in T_{1}$, then $\$_{2}=l\left(\$_{1}\right)$ and if $\$_{1} \notin T_{1}$, then $\$_{2}=0$. Consider $l_{1}$ being a function from the carrier of $X$ into $\mathbb{R}$ such that for every object $x$ such that $x \in$ the carrier of $X$ holds $\mathcal{Q}\left[x, l_{1}(x)\right]$ from [10, Sch. 1].

(8) Suppose $A$ misses $B$. Then there exists a linear combination $l_{1}$ of $A$ and there exists a linear combination $l_{2}$ of $B$ such that the support of $l=\left(\right.$ the support of $\left.l_{1}\right) \cup\left(\right.$ the support of $\left.l_{2}\right)$ and $l=l_{1}+l_{2}$ and the support of $l_{1}=($ the support of $l) \backslash B$ and the support of $l_{2}=($ the support of $l) \backslash A$. The theorem is a consequence of (7).

Now we state the propositions:

(9) Let us consider a real linear space $X$, subsets $A, B$ of $X$, a linear combination $l_{1}$ of $A$, and a linear combination $l_{2}$ of $B$. Suppose $\operatorname{rng} l_{1} \subseteq \mathbb{Q}$ and $\operatorname{rng} l_{2} \subseteq \mathbb{Q}$ and $A$ misses $B$. Then there exists a linear combination $l$ of $A \cup B$ such that

(i) the support of $l=\left(\right.$ the support of $\left.l_{1}\right) \cup\left(\right.$ the support of $l_{2}$ ), and

(ii) $\operatorname{rng} l \subseteq \mathbb{Q}$, and

(iii) $\sum l=\sum l_{1}+\sum l_{2}$.

The theorem is a consequence of (6).

(10) Let us consider a real linear space $X$, subsets $A, B$ of $X$, and a linear combination $l$ of $A \cup B$. Suppose $\operatorname{rng} l \subseteq \mathbb{Q}$ and $A$ misses $B$. Then there exists a linear combination $l_{1}$ of $A$ and there exists a linear combination $l_{2}$ of $B$ such that $\operatorname{rng} l_{1} \subseteq \mathbb{Q}$ and $\operatorname{rng} l_{2} \subseteq \mathbb{Q}$ and $\sum l=\sum l_{1}+\sum l_{2}$. The theorem is a consequence of $(8)$.

(11) Let us consider a real linear space $X$, and finite subsets $A, B$ of $X$. Suppose $A$ misses $B$. Then $\operatorname{Sums}_{\mathbb{Q}} A+\operatorname{Sums}_{\mathbb{Q}} B=\operatorname{Sums}_{\mathbb{Q}}(A \cup B)$. The theorem is a consequence of (9) and (10). 
Let $X$ be a real linear space and $A$ be a finite subset of $X$. Observe that Sums $_{\mathbb{Q}} A$ is countable.

Now we state the proposition:

(12) Let us consider a real linear space $X$, a sequence $x$ of $X$, and a finite subset $A$ of $X$. Suppose $A \subseteq \operatorname{rng} x$. Then there exists a natural number $n$ such that $A \subseteq \operatorname{rng}\left(x \mid \mathbb{Z}_{n}\right)$.

Proof: Define $\mathcal{P}$ [natural number] $\equiv$ for every finite subset $A$ of $X$ such that $\overline{\bar{A}}=\$_{1}$ and $A \subseteq \operatorname{rng} x$ there exists a natural number $n$ such that $A \subseteq \operatorname{rng}\left(x \mid \mathbb{Z}_{n}\right) . \mathcal{P}[0]$. For every natural number $k$ such that $\mathcal{P}[k]$ holds $\mathcal{P}[k+1]$ by [3, (44)], [12, (31)], [3, (42)], [10, (11)]. For every natural number $k, \mathcal{P}[k]$ from [5, Sch. 2].

Let $X$ be a real linear space and $x$ be a sequence of $X$. One can verify that Sums $\mathbb{Q}$ rng $x$ is countable.

Now we state the propositions:

(13) Let us consider a real normed space $X$, and a sequence $x$ of $X$. Then Sums $_{\mathbb{Q}}$ rng $x$ is a subset of the carrier of NLin rng $x$.

Proof: Set $D=$ Sums $\mathbb{Q} \operatorname{rng} x$. For every object $z$ such that $z \in D$ holds $z \in$ the carrier of NLin rng $x$ by [30, (14)].

(14) Let us consider a real normed space $X$, and a subset $Y$ of $X$. Then

(i) the carrier of NLin $Y \subseteq$ the carrier of $\operatorname{ClNLin}(Y)$, and

(ii) there exists a subset $Z$ of $X$ such that $Z=$ the carrier of NLin $Y$ and $\bar{Z}=$ the carrier of $\operatorname{ClNLin}(Y)$.

(15) Let us consider a real normed space $X$, and a sequence $x$ of $X$. Then Sums $\mathbb{Q} \operatorname{rng} x$ is a countable subset of the carrier of ClNLin( $(\operatorname{nng} x)$. The theorem is a consequence of (13) and (14).

(16) Let us consider real numbers $z$, e. Suppose $0<e$. Then there exists an element $q$ of $\mathbb{Q}$ such that

(i) $q \neq 0$, and

(ii) $|z-q|<e$.

(17) Let us consider a real normed space $X$, a point $w$ of $X$, a real number $e$, and a linear combination $l$ of $\{w\}$. Suppose $0<e$. Then there exists a linear combination $m$ of $\{w\}$ such that

(i) the support of $m=$ the support of $l$, and

(ii) $\operatorname{rng} m \subseteq \mathbb{Q}$, and

(iii) $\left\|\sum l-\sum m\right\|<e$.

The theorem is a consequence of (16). 
(18) Let us consider a real normed space $X$, a subset $A$ of $X$, a real number $e$, and a linear combination $l$ of $A$. Suppose $0<e$. Then there exists a linear combination $m$ of $A$ such that

(i) the support of $m=$ the support of $l$, and

(ii) $\operatorname{rng} m \subseteq \mathbb{Q}$, and

(iii) $\left\|\sum l-\sum m\right\|<e$.

Proof: Define $\mathcal{P}$ [natural number] $\equiv$ for every real number $e$ for every linear combination $l$ of $A$ such that $0<e$ and $\overline{\overline{\text { the support of } l}}=\$_{1}$ there exists a linear combination $m$ of $A$ such that the support of $m=$ the support of $l$ and $\operatorname{rng} m \subseteq \mathbb{Q}$ and $\left\|\sum l-\sum m\right\|<e . \mathcal{P}[0]$ by [29, (34), (44), (42)], [30, (2)]. For every natural number $k$ such that $\mathcal{P}[k]$ holds $\mathcal{P}[k+1]$ by [3, (44)], [12, (31)], [3, (42)], (8). For every natural number $k$, $\mathcal{P}[k]$ from [5, Sch. 2].

Let us consider a real normed space $X$ and a sequence $x$ of $X$. Now we state the propositions:

(19) Sums $\operatorname{Se}_{\mathbb{Q}} \operatorname{rng} x$ is a dense subset of the carrier of NLin rng $x$.

(20) Sums $\mathbb{Q} \operatorname{rng} x$ is a dense subset of the carrier of ClNLin $(\operatorname{rng} x)$.

Now we state the proposition:

(21) Let us consider a real normed space $X$. Suppose there exists a subset $D$ of the carrier of $X$ such that $D$ is dense and countable. Then $X$ is separable.

\section{Basic Properties of Separable Spaces}

Let $X$ be a real normed space and $x$ be a sequence of $X$. Let us observe that $\mathrm{ClNLin}(\operatorname{rng} x)$ is separable.

Now we state the propositions:

(22) Let us consider a real normed space $X$, a real normed subspace $Y$ of $X$, and a Lipschitzian linear functional $L$ in $X$. Then $L \uparrow($ the carrier of $Y$ ) is a Lipschitzian linear functional in $Y$.

Proof: Set $Y_{1}=$ the carrier of $Y$. Reconsider $L_{1}=L\left\lceil Y_{1}\right.$ as a functional in $Y . L_{1}$ is additive by [9, (49)], [19, (28)]. $L_{1}$ is homogeneous by [9, (49)], [19, (28)]. Consider $K$ being a real number such that $0 \leqslant K$ and for every point $x$ of $X,|L(x)| \leqslant K \cdot\|x\|$. For every point $x$ of $Y,\left|L_{1}(x)\right| \leqslant K \cdot\|x\|$ by [19, (28)], [9, (49)].

(23) Let us consider real normed spaces $X, Y$, a subset $A$ of $X$, a subset $B$ of $Y$, and a Lipschitzian linear operator $L$ from $X$ into $Y$. Suppose $L$ is isomorphism and $B=L^{\circ} A$. Then $A$ is dense if and only if $B$ is dense. 
(24) Let us consider real normed spaces $X, Y$. Suppose there exists a Lipschitzian linear operator $L$ from $X$ into $Y$ such that $L$ is isomorphism. Then $X$ is separable if and only if $Y$ is separable. The theorem is a consequence of $(23)$.

(25) Let us consider a real normed space $X$. Suppose $X$ is non trivial and reflexive. Then $X$ is separable if and only if $\operatorname{DualSp}(\operatorname{DualSp}(X))$ is separable. The theorem is a consequence of (24).

\section{Completeness and Reflexivity of Sublinear Normed Spaces}

Now we state the proposition:

(26) Let us consider a real normed space $X$, and subsets $Y, Z$ of $X$. Suppose $Z=$ the carrier of $\operatorname{Lin}(Y)$. Then the carrier of $\operatorname{Lin}(Z)=Z$.

Let us consider a real Banach space $X$ and a subset $Y$ of $X$. Now we state the propositions:

(27) There exists a subset $Z$ of $X$ such that

(i) $Z=$ the carrier of $\operatorname{Lin}(Y)$, and

(ii) $\operatorname{ClNLin}(Y)=\operatorname{NLin} \bar{Z}$, and

(iii) $\bar{Z}$ is linearly closed, and

(iv) $\bar{Z} \neq \emptyset$.

(28) $\mathrm{ClNLin}(Y)$ is a real Banach space. The theorem is a consequence of (27).

(29) If $X$ is reflexive, then $\operatorname{ClNLin}(Y)$ is reflexive. The theorem is a consequence of (27).

ACKNowledgement: We would like to thank Keiko Narita and Yasunari Shidama.

\section{REFERENCES}

[1] Jonathan Backer, Piotr Rudnicki, and Christoph Schwarzweller. Ring ideals Formalized Mathematics, 9(3):565-582, 2001.

[2] Grzegorz Bancerek. Cardinal numbers Formalized Mathematics, 1(2):377-382, 1990.

[3] Grzegorz Bancerek. Cardinal arithmetics Formalized Mathematics, 1(3):543-547, 1990.

[4] Grzegorz Bancerek. König's theorem. Formalized Mathematics, 1(3):589-593, 1990.

[5] Grzegorz Bancerek. The fundamental properties of natural numbers Formalized Mathematics, 1(1):41-46, 1990.

[6] Grzegorz Bancerek. The ordinal numbers. Formalized Mathematics, 1(1):91-96, 1990.

[7] Nicolas Bourbaki. Topological vector spaces: Chapters 1-5. Springer, 1981.

[8] Czesław Byliński. The complex numbers. Formalized Mathematics, 1(3):507-513, 1990.

[9] Czesław Byliński. Functions and their basic properties Formalized Mathematics, 1(1): 55-65, 1990. 
[10] Czesław Byliński. Functions from a set to a set Formalized Mathematics, 1(1):153-164, 1990.

[11] Czesław Byliński. Partial functions. Formalized Mathematics, 1(2):357-367, 1990.

[12] Czesław Byliński. Some basic properties of sets Formalized Mathematics, 1(1):47-53, 1990.

[13] Agata Darmochwał. Finite sets. Formalized Mathematics, 1(1):165-167, 1990.

[14] N. J. Dunford and T. Schwartz. Linear operators I. Interscience Publ., 1958.

[15] Noboru Endou, Yasunari Shidama, and Katsumasa Okamura. Baire's category theorem and some spaces generated from real normed space. Formalized Mathematics, 14(4): 213-219, 2006. doi 10.2478/v10037-006-0024-x.

[16] Andrey Kolmogorov and Sergei Fomin. Elements of the Theory of Functions and Functional Analysis [Two Volumes in One]. Martino Fine Books, 2012.

[17] Andrzej Kondracki. Basic properties of rational numbers Formalized Mathematics, 1(5): 841-845, 1990.

[18] Eugeniusz Kusak, Wojciech Leończuk, and Michał Muzalewski. Abelian groups, fields and vector spaces. Formalized Mathematics, 1(2):335-342, 1990.

[19] Kazuhisa Nakasho, Yuichi Futa, and Yasunari Shidama. Topological properties of real normed space. Formalized Mathematics, 22(3):209-223, 2014. doi:10.2478/forma-20140024 .

[20] Keiko Narita, Noboru Endou, and Yasunari Shidama. Dual spaces and Hahn-Banach theorem. Formalized Mathematics, 22(1):69-77, 2014. doi 10.2478/forma-2014-0007.

[21] Keiko Narita, Noboru Endou, and Yasunari Shidama. Bidual spaces and reflexivity of real normed spaces. Formalized Mathematics, 22(4):303-311, 2014. doi 10.2478/forma2014-0030.

[22] Bogdan Nowak and Andrzej Trybulec. Hahn-Banach theorem Formalized Mathematics, 4(1):29-34, 1993.

[23] Beata Padlewska and Agata Darmochwał. Topological spaces and continuous functions Formalized Mathematics, 1(1):223-230, 1990.

[24] Jan Popiołek. Real normed space Formalized Mathematics, 2(1):111-115, 1991.

[25] Yasunari Shidama. Banach space of bounded linear operators Formalized Mathematics, 12(1):39-48, 2004.

[26] Andrzej Trybulec. Domains and their Cartesian products. Formalized Mathematics, 1(1): 115-122, 1990.

[27] Wojciech A. Trybulec. Subspaces and cosets of subspaces in real linear space Formalized Mathematics, 1(2):297-301, 1990.

[28] Wojciech A. Trybulec. Vectors in real linear space. Formalized Mathematics, 1(2):291-296, 1990.

[29] Wojciech A. Trybulec. Linear combinations in real linear space Formalized Mathematics, $1(\mathbf{3}): 581-588,1990$.

[30] Wojciech A. Trybulec. Basis of real linear space. Formalized Mathematics, 1(5):847-850, 1990.

[31] Zinaida Trybulec. Properties of subsets Formalized Mathematics, 1(1):67-71, 1990.

[32] Edmund Woronowicz. Relations and their basic properties. Formalized Mathematics, 1 (1):73-83, 1990.

[33] Edmund Woronowicz. Relations defined on sets. Formalized Mathematics, 1(1):181-186, 1990.

[34] Kosaku Yoshida. Functional Analysis. Springer, 1980.

Received February 26, 2015 